\title{
Author Index Vol. 15, No. 3-5, 1993
}

\section{$(\mathrm{A})=$ Abstracts}

Baarsma,R. 240 [Bachelard, H. 207 f Badar-GofTer, R. 207

Bates, T.E. 174

Behar.K.L. 181 pen-Yoseph, 0. 207, 380 (A)

Boatright,R. 320

Boer, J. de 240 Boxer, P.A. 380(A) ! Brand, A. 289 prown,M.C. 378(A) [Bryan, G.K. 271 feutterworth, R.F. 313

Clark, J.B. 174 [Cullingford, T. 174

E)aikhin,y. 343 Denton, R.M. 165 Driscoll, B.F. 282,299

punn,J.R 378(A)

Edmond, J. 151 Erecinska, M. 343

(Fonnum, F. 351

Gabryel, B. 379 (A), 380 (A) 
Klimaszewski, A. 216

Korf,J. 240

Kozlowski,A. 379 (A), 380 (A)

Lai,J.C.K. 181 Land,J.M. 174 Larsson, O. 367 Lehmann,J.C. 330 Leibfritz,D. 289 Leo, G.C. 282 Lin,Z.-P. 343 Lund, P. 156

McCormack, J.G. 165 McKenna, M.C. 226,320 Magistretti, P. J. 306 Malecki,A. 379(A), 380(A) Manos,P. 271 Martin, J.-L. 306 Morris, P. 207 Miiller,T.B. 351

Nabetani,M. 378(A) Nelson, D. 343 Nissim, I. 343

Okada,Y. 378(A) Okken,A. 240 
Radda,G.K. 378(A) Richter-Landsberg, C. 289 Rodriguez de Lores Amaiz, G. 379 (A) Ross,B.D. 380(A)

Schousboe, A. 351,359,367 Shank, R.P. 282,330 SokoIoff,L. 194 Sonnewald, U. 351,359,367 Sorg,0. 306 Stevenson, J.H. 226,320

Thatcher, N. 207 Tildon, J.T. 154,226,233, 320 Trzeciak, H.I. 379(A), 380(A) Tsacopoulos, M. 336

Tsao,J.W. 378(A) Tsuji,M. 261

Unsgard,G. 351

Venema,K. 240 Veuthey,A.-L. 336

Wallimann, T. 249,261 Walz,W. 216

Westergaard, N. 351, 359, 367 Williamson, D.H. 156

passel,B. 351 Hemmer,W. 249,261 Hertz, L. 351,359,367 poltzman,D. 247,261 Huang, R. 359,367 B|uang,S. 320

Huang, Y. 233

Kapkov,D. 330 Kaufman, E3. 282,299 
Paramananthan, N. 378(A) Paterson, LA. 216 Pellerin,L. 306 Peng,L. 359,367 Perry, V.H. 378(A) Petersen, S.B.

351,359,367 Pleasure, D. 343 Pudelko,A. 379(A), 380(A) 
Yu,N. 306 Yudkoff, M. 280,343

Zielke,C.L. 233 Zielke, H.R. 233

381 